\title{
Non-Procrustean pathways for complex generic drugs development
}

\author{
Rodrigo Cristofoletti*.1, Stephan Schmidt ${ }^{2}$ \& Andrea Diniz ${ }^{3}$ \\ ${ }^{1}$ Brazilian Health Surveillance Agency (ANVISA), Division of Therapeutic Equivalence, Brasilia, Brazil \\ ${ }^{2}$ Center for Pharmacometrics \& Systems Pharmacology, University of Florida, Orlando, FL, USA \\ ${ }^{3}$ Departamento de Farmacia, Universidade Estadual de Maringá, Maringá, Brazil \\ *Author for correspondence: Tel.: +55 613462 5589; rodrigocrsistofol@gmail.com
}

"using integrative approaches like the model-informed drug development, which includes PKPD models, mechanistic absorption models, physiologically based pharmacokinetic (PBPK) models, systems pharmacology and quantitative risk modeling) may be useful to improve drug development knowledge management and decision-making on a non-Procrustean way."

First draft submitted: 28 June 2018; Accepted for publication: 12 July 2018; Published online: 7 September 2018

Keywords: bioequivalence $\bullet$ complex generics $\bullet$ model-informed drug development $\bullet$ modeling and simulation

\section{Bioequivalence for complex generics}

Overall, legal texts tend to be abstruse and, even if one is proficient in Legalese, their interpretation may be dubious offering more than one equally plausible path forward. The situation is somewhat more straightforward in the pharmaceutical arena, not only in terms of language but also for setting clear regulatory standards. The downside of this strategy is the overuse of one-size-fits-all (OSFA) approaches. Even though OSFA approaches ensure harmonization throughout the regulatory decision-making process, they may not be adequate for all the cases. Paraphrasing the famous Albert Einstein quote of the Occam's razor principle (i.e., problem-solving/logical principle that, when you have two competing theories that make exactly the same predictions, the simpler one is the better, also known as the 'law of parsimony'), we may state that "regulatory guidelines should be made as simple as possible but not any simpler". In fact, during a 2006 Advisory Committee for Pharmaceutical Science meeting at the US FDA, Leslie Benet declared the then current OSFA-based bioequivalence (BE) guidelines to be Procrustean [1]. In Greek mythology, Procrustes was a robber who killed his victims in the most cruel and unusual way. He made them lie on an iron bed and would cut off body parts of the people who were too large or stretch people who were too short [2]. According to the Merriam-Webster dictionary, Procrustean takes no account of individual differences, but makes everyone the same. However, it is worth noting that the regulatory paradigm in the realm of generic drugs has advanced toward non-Procrustean approaches, for example, with the Biopharmaceutics Classification System-based biowaiver decisions, as well as reference-scaled average BE for highly variable drugs, keeping the pace with the modern era of individualized and precision medicine [3,4].

Demonstration of therapeutic equivalence for so-called 'complex generics' however remains challenging, as recently stated by the FDA Commissioner [5]. In order to approach these more complex scenarios, respective cases can be subdivided into four different categories: complex routes of delivery; complex formulations; complex drug substances and complex drug-device combination.

First, there are complex routes of delivery (e.g., locally acting ophthalmic drugs). Due to the very low to undetectable levels of drug in the systemic circulation following the administration of ophthalmics, traditional average BE studies, focusing on systemic exposure metrics to compare test $(T)$ and reference (R) formulations, is not adequate. In this case, the FDA has recommended two alternative approaches: in vitro or in vivo patient studies. If $\mathrm{T}$ and $\mathrm{R}$ formulations are qualitatively (Q1) and quantitatively (Q2) the same, extensive comparative physicochemical characterization in vitro may be enough to declare BE. For example, particle size distribution should be compared using population BE, a concept that was intensely debated throughout the 1990s, but has not been formally incorporated in the legal framework related to generics until recently. Alternatively, for 
non-Q1/Q2 products containing ophthalmic corticosteroids, pharmacokinetic (PK) studies in aqueous humor of patients undergoing indicated cataract surgery is recommended. Since noncompartmental analysis is rather sensitive to the number of sampling time points, alternative statistical approaches intended to characterize the time course of systemic drug exposure (including utilization of bootstrapping to estimate respective standard deviations) or population-based models are required $[6,7]$. For drugs intended to treat chronic open-angle glaucoma, ocular hypertension or pain and inflammation associated with cataract surgery, BE is established based on the clinical end points, for instance the difference in intraocular pressure and proportion of subjects with cure at postop day 14 [6].

The second category encompasses complex formulations (e.g., liposomes). In this case, similar systemic exposure, in terms of total drug content, does not necessarily mean similarity in tissue exposure. In fact, formulation-dependent target site distribution has already been reported in both preclinical and clinical situations for parenteral products containing pegylated liposomal doxorubicin or paclitaxel protein-bound particles for injectable suspension $[8,9]$. Thus, the most challenging issue in demonstrating BE between complex formulations is to identify the most therapeutically relevant surrogate to bridge the gap between systemic exposure and target site disposition [3]. Consequently, BE demonstration generally implies analyzing the total and unbound or free and encapsulated drug concentrations. Additionally, comparison of in vitro particle/liposome size distribution using the population $\mathrm{BE}$ approach and demonstration of compositional equivalence are also recommended.

For complex drug substances (e.g., synthetic peptides and copolymers), the main issue does not arise from BE assessment per se but from the demonstration of absence of differences between active pharmaceutical ingredients (API) by different manufacturers. Glatiramer acetate is a classical representative of this category. While its formulation is rather simple (e.g., prefilled syringe containing $1 \mathrm{ml}$ solution with $20 \mathrm{mg}$ of glatiramer acetate and $40 \mathrm{mg}$ of mannitol), showing the mixture of peptide copolymers is the same in $\mathrm{T}$ and $\mathrm{R}$ formulations is rather challenging. The state-of-art knowledge advocates applying a four-stage criteria focusing on demonstrations of: equivalence of fundamental reaction scheme; equivalence of physicochemical properties including compositions; equivalence of structural signatures for polymerization and depolymerization and equivalence of biological assay results. Even though this is a synthetic drug, regulatory criteria are similar to the head-to-head comparison currently used to evaluate biosimilars. Interestingly, the biological assay can serve as a confirmatory test of equivalence and provide complementary confirmation of API sameness. In other words, similar pharmacodynamic (PD) response is ultimately used to infer back similar binding/affinity to the target and thus, similar API structure/composition [10]. Other representatives of this category are iron-based products stabilized by a complex carbohydrate, which leads to nano-sized colloidal structure. In this case, the major challenge is once more to identify the most relevant surrogate on which BE would be based. Differently from the rationale for complex formulations, measuring solely drugbound iron in serum (e.g., difference in AUC between total iron and transferrin-bound iron) is generally chosen since it represents the amount of iron available for biological processes [3]. Notwithstanding, similar PK profiles do not discriminate the in vivo fate of drug-bound iron since it may be cleared by reticuloendothelial system uptake or by simply in vivo release of iron. Thus, it is not sufficient for the assurance of comparability of the resulting toxicological and pharmacological effects of these products. Consequently, comprehensive product characterization in vitro and tissue bio-distribution studies in animals are recommended by the regulatory authorities [3,11].

Last but not the least, there are the complex drug-device combinations (e.g., metered-dose inhalers, dry powder inhalers or nasal sprays). Drug delivery characteristics of orally inhaled products (OIP) is rather dependent on the respective device, which should be similar enough without infringing on the innovator company's patent. Changing the device may be as critical as changing the amount of surfactant in an oral formulation containing a poorly soluble drug. In the case of OIP, another issue should be considered: systemic exposure is downstream of the site of action and, therefore at best, equivalent systemic exposure means equivalence in terms of safety only. Furthermore, one should consider the confluence of two routes of absorption after administering OIPs. A considerable drug fraction gets no further than the oropharynx and is swallowed. This swallowed portion may still be absorbed from the GI tract acting as a confounding factor when using systemic exposure to infer about drug delivery and local lung deposition. Consequently, the traditional PK-based OSFA approach is not suitable to address the BE of OIP. Indeed, a combination of in vitro, PK and PD studies are required. Importantly, when PD biomarkers are used directly to assess $\mathrm{BE}$, doses need to be used that lie in the steep part of the dose-response curve for efficacy; otherwise, the biopharmaceutical aspect of the BE might be neglected [12]. 


\section{Conclusion}

The above-listed examples of complex drugs comprise high-cost medicines and, generally, the branded drugs face no market competition due to the difficulties surrounding generic development, even if the patents have already expired. Therefore, developing scientifically sound, product-specific requirements is imperative. In this regard, regulatory authorities have announced new policies, focusing on adopting more rigorous and sophisticated science, including quantitative methods and computational modeling in drug development, evaluation and review [13]. In fact, using integrative approaches like the model-informed drug development, which includes PKPD models, mechanistic absorption models, physiologically based pharmacokinetic (PBPK) models, systems pharmacology and quantitative risk modeling) may be useful to improve drug development knowledge management and decision-making on a non-Procrustean way.

\section{Financial \& competing interests disclosure}

The authors have no relevant affiliations or financial involvement with any organization or entity with a financial interest in or financial conflict with the subject matter or materials discussed in the manuscript. This includes employment, consultancies, honoraria, stock ownership or options, expert testimony, grants or patents received or pending, or royalties.

No writing assistance was utilized in the production of this manuscript.

\section{References}

1 Benet L. Why highly variable drugs are safer, FDA Advisory Committee for Pharmaceutical Sciences and Clinical Pharmacology meeting transcript. (2006). http://slideplayer.com/slide/9823715/

2 Procrustes. Myths and Legends of the World. (2018). www.encyclopedia.com

3 Zhang X, Zheng N, Lionberger RA, Yu LX. Innovative approaches for demonstration of bioequivalence: the US FDA perspective. Ther Deliv. 4(6), 725-740 (2013).

4 Lesko LJ, Schmidt S. Individualization of drug therapy: history, present state, and opportunities for the future. Clin. Pharmacol. Ther 92(4), 458-466 (2012).

5 Gottlieb, S. Reducing the hurdles for complex generic drug development. (2018). https://blogs.fda.gov/fdavoice/index.php/2017/10/reducing-the-hurdles-for-complex-generic-drug-development/

6 Choi SH, Lionberger RA. Clinical, pharmacokinetic, and in vitro studies to support bioequivalence of ophthalmic drug products. AAPS J. 18(4), 1032-1038 (2016).

7 Ducharme M. Strengths and weaknesses of population PK analyses for the assessment of bioequivalence of complex and locally acting products. In: Leveraging Quantitative Methods and Modeling to Modernize Generic Drug Development and Review; Public Workshop, 2-3 October 2017. (2017). www.fda.gov/downloads/Drugs/NewsEvents/UCM582132.pdf

8 Cui J, Li C, Guo W et al. Direct comparison of two pegylated liposomal doxorubicin formulations: is AUC predictive for toxicity and efficacy? J. Control. Rel. 118(2), 204-215 (2007).

9 Sparreboom A, van Zuylen L, Brouwer E et al. Cremophor EL-mediated alteration of paclitaxel distribution in human blood: clinical pharmacokinetic implications. Cancer Res. 59(7), 1454-1457 (1999).

10 Anderson J, Bell C, Bishop J et al. Demonstration of equivalence of a generic glatiramer acetate (Glatopa ${ }^{\text {TM }}$. J. Neurol. Sci. 359(1-2), 24-34 (2015).

11 European Medicines Agency (EMA). Reflection paper on the data requirements for intravenous iron-based nano-colloidal products developed with reference to an innovator medicinal product. (2015). www.ema.europa.eu/docs/en_GB/document_library/Scientific_guideline/2015/03/WC500184922.pdf

12 Hochhaus G, Horhota S, Hendeles L, Suarez S, Rebello J. Pharmacokinetics of orally inhaled drug products. AAPS J. 17(3), 769-775 (2015).

13 United State Food and Drug Administration (US-FDA). Generic Drug User Fee Amendments (GDUFA) - Pre-ANDA program for complex products in GDUFA II. (2017). www.fda.gov/ForIndustry/UserFees/GenericDrugUserFees/ucm580458.htm 
